\title{
Score for Predicting Active Cancer in Patients with Ischemic Stroke: A Retrospective Study
}

\author{
Jiwei Jiang $\mathbb{D}^{1},{ }^{1}$ Xiuli Shang $\mathbb{D}^{2},{ }^{2}$ Jinming Zhao, ${ }^{3}$ Meihui Cao, ${ }^{4}$ Jirui Wang, ${ }^{2}$ Runzhi Li $\mathbb{D}^{1}$ \\ Yanli Wang ${ }^{D}{ }^{1}$ and Jun $X u{ }^{1}$ \\ ${ }^{1}$ Department of Neurology, Beijing Tiantan Hospital, Capital Medical University, Beijing, China \\ ${ }^{2}$ Department of Neurology, The First Affiliated Hospital, China Medical University, Shenyang, China \\ ${ }^{3}$ Department of Pathology, The First Affiliated Hospital and College of Basic Medical Sciences, China Medical University, China \\ ${ }^{4}$ Department of Oncology, Cancer Hospital of China Medical University, Liaoning Cancer Hospital \& Institute, China
}

Correspondence should be addressed to Jun Xu; neurojun@126.com

Received 3 March 2021; Revised 1 April 2021; Accepted 18 May 2021; Published 25 May 2021

Academic Editor: Yuzhen Xu

Copyright (c) 2021 Jiwei Jiang et al. This is an open access article distributed under the Creative Commons Attribution License, which permits unrestricted use, distribution, and reproduction in any medium, provided the original work is properly cited.

Background. We aimed to examine the differences of clinical characteristics between patients with ischemic stroke with active cancer and those without cancer to develop a clinical score for predicting the presence of occult cancer in patients with ischemic stroke. Methods. This retrospective study enrolled consecutive adult patients with acute ischemic stroke who were admitted to our department between December 2017 and January 2019. The demographic, clinical, laboratory, and neuroimaging characteristics were compared between patients with ischemic stroke with active cancer and those without cancer. Multivariate analysis was performed to identify independent factors associated with active cancer. Subsequently, a predictive score was developed using the areas under the receiver operating characteristic curves based on these independent factors. Finally, Bayesian decision theory was applied to calculate the posterior probability of active cancer for finding the best scoring system. Results. Fifty-three (6.63\%) of 799 patients with ischemic stroke had active cancer. The absence of a history of hyperlipidemia (odds ratio $(\mathrm{OR})=0.17,95 \%$ confidence interval $(\mathrm{CI}): 0.06-0.48, P<0.01)$, elevated serum fibrinogen $(\mathrm{OR}=1.72,95 \% \mathrm{CI}$ : $1.33-$ 2.22, $P<0.01)$ and $\mathrm{D}$-dimer levels $(\mathrm{OR}=1.43,95 \% \mathrm{CI}: 1.24-1.64, P<0.01)$, and stroke of undetermined etiology $(\mathrm{OR}=22.87$, 95\% CI: 9.91-52.78, $P<0.01)$ were independently associated with active cancer. A clinical score based on the absence of hyperlipidemia, serum fibrinogen level of $\geq 4.00 \mathrm{~g} / \mathrm{L}$, and D-dimer level of $\geq 2.00 \mu \mathrm{g} / \mathrm{mL}$ predicted active cancer with an area under the curve of 0.83 (95\% CI: $0.77-0.89, P<0.01$ ). The probability of active cancer was $59 \%$ at a supposed prevalence of $6.63 \%$, if all three independent factors were present in a patient with ischemic stroke. Conclusions. We devised a clinical score to predict active cancer in patients with ischemic stroke based on the absence of a history of hyperlipidemia and elevated serum $\mathrm{D}$ dimer and fibrinogen levels. The use of this score may allow for early intervention. Further research is needed to confirm the implementation of this score in clinical settings.

\section{Introduction}

Malignant tumors and stroke are the most common causes of disability and mortality worldwide [1]. The concomitance of both conditions has serious repercussions on the quality of life, with a substantial increase in the socioeconomic burden at both the individual and societal levels. Previous studies have reported that approximately $15 \%$ of patients with cancer are at risk of developing ischemic stroke (IS) later in life [2], and $10 \%$ of patients hospitalized with IS could have cancer as a comorbidity [3]. Sometimes, IS could even represent the initial manifestation of occult cancer [4]. A systematic review revealed that the short-term risk of stroke was considerably increased after a new diagnosis of a solid tumor, and correspondingly, the probability of diagnosing cancer was significantly high in the first few months after a stroke [5]; moreover, the risk of stroke varies with the type of cancer, histopathological features, and stage [6]. This 
evidence demonstrates a close association between cancer and stroke, revealing an opportunity for occult cancer screening in specific populations, such as patients with cryptogenic stroke. Thus, discerning occult malignancies at an early stage in patients with IS and treatment with appropriate interventions could help improve the chances of survival and functional outcomes [7].

However, there is no consensus on the characteristics of patients with IS at a higher risk of developing malignancies, despite the increasing interest in the relationship between IS and cancer $[6,8]$. Some studies have shown that higher rates of stroke of undetermined etiology (SUE), elevated C-reactive protein (CRP) and D-dimer levels, and lesions involving multiple vascular territories as shown on diffusion-weighted imaging (DWI) are relatively specific characteristics of patients with cancer-associated IS [9-11]. However, other studies did not find significant differences in these stroke-related characteristics between IS patients with and without cancer [12-14]. Currently, research focusing on the appropriate time and approach for screening cancer in patients with IS is lacking. To the best of our knowledge, only one study has reported a score for predicting occult malignancies in patients with IS; however, in terms of overall predictive strength, that score had an area under the curve (AUC) of 0.73 [15]. Therefore, we conducted a retrospective study to identify the differences in clinical, laboratory, and neuroimaging characteristics between IS patients with active cancer or without cancer and to develop a more reliable, clinically relevant predictive score for cancer screening in patients with IS.

\section{Materials and Methods}

2.1. Patient Selection and Data Collection. This retrospective study enrolled consecutive patients with acute IS from the Department of Neurology at the First Affiliated Hospital of China Medical University, a comprehensive academic hospital in a large urban area, between December 2017 and January 2019. The inclusion criteria were as follows: (1) age $\geq 18$ years, (2) IS that met the Baltimore-Washington Cooperative Young Study Criteria, (3) neurologic deficit lasting longer than $24 \mathrm{~h}$, and (4) computed tomography (CT) or magnetic resonance imaging (MRI) scans depicting infarctions consistent with clinical findings [16]. Patients were excluded from the study if they (1) lacked information regarding the etiological examination for stroke, including data from Holter electrocardiography (ECG), transthoracic or transesophageal echocardiography, magnetic resonance angiography (MRA), CT angiography (CTA), or Doppler ultrasonography; (2) were diagnosed with transient ischemic attack (TIA) or cerebral hemorrhage; (3) had a previous history of brain tumor, cerebral metastases, or intracranial surgery; and (4) had indications of inactive cancer (patients with IS and cancer that did not meet the description of active cancer) or hematological malignancies. Active cancer was defined as the diagnosis of cancer or administration of cancer treatment within the past 6 months or the metastasis or recurrence of known cancers $[17,18]$. Patients with IS and active cancer were categorized into the cancer group, while those without cancer were assigned to the control group.
2.2. Clinical Assessment. Trained personnel reviewed the patients' demographic data (age, sex, and medical history); vascular risk factors (hypertension: previous diagnosis and treatment or blood pressure $\geq 140 / 90 \mathrm{mmHg}$ ); diabetes mellitus (previous diagnosis and treatment or fasting glucose level of $\geq 7.00 \mathrm{mmol} / \mathrm{L}$ ); hyperlipidemia (previous diagnosis and treatment; fasting total serum cholesterol level of $\geq 5.72 \mathrm{mmol} / \mathrm{L}$, triglyceride level of $\geq 1.7 \mathrm{mmol} / \mathrm{L}$, or low-density lipoprotein level of $\geq 3.64 \mathrm{mmol} / \mathrm{L}$ ); atrial fibrillation (previous diagnosis and treatment or suggestive ECG findings); history of coronary heart disease, IS, or TIA; obesity (body mass index $\geq 28 \mathrm{~kg} / \mathrm{m}^{2}$ ); current smoking habits; stroke severity on admission (based on the National Institute of Health Stroke Scale (NIHSS) score) [19]; and results of diagnostic investigations. Two boardcertified neurologists consensually determined the stroke etiology as large-artery atherosclerosis (LAA), cardioembolism (CE), small vessel occlusion (SVO), stroke of other determined etiology (SOE), or SUE, according to the Trial of Org 10172 in Acute Stroke Treatment (TOAST) criteria [20].

Routine investigations included neuroimaging (MRI, especially contrast-enhanced MRI and DWI, and angiography, including MRA, CTA, and Doppler ultrasonography), ECG or $24 \mathrm{~h}$ ECG, and transthoracic or transesophageal echocardiography. The presence of acute multiple cerebral infarctions (AMCI) was determined based on DWI findings, and AMCI were defined as multiple acute infarcts, either in the bilateral anterior or posterior circulation or simultaneously in the anterior and posterior circulations, suggesting an embolic etiology. Data on routine laboratory parameters, including serum platelet counts and hemoglobin, fibrinogen, D-dimer, CRP, lipid, and glucose levels, were also obtained. The diagnosis of cancer was confirmed based on histopathological evidence and the oncologist's opinion. Furthermore, oncological records were carefully reviewed for cancerspecific details, including tumor type, histology, and stage.

2.3. Statistical Analysis. All statistical analyses were performed using SPSS 22.0 statistical software (SPSS Inc., Chicago, IL, USA). Categorical variables are presented as the total number $(n)$ and percentage (\%) per group, and the $\chi^{2}$ or Fisher's exact test was used to assess statistical differences. The mean and standard deviations (SDs) were calculated for continuous variables with normal distribution, while the median and interquartile range (IQR) were used for continuous variables lacking normal distribution. Similarly, the Student $t$-test was used for normally distributed data. The Mann-Whitney $U$ test was used for data without normal distribution. Risk factors $(P \leq 0.05)$ were further analyzed using univariate and multivariate logistic regression. Risk factors independently associated with active cancer were included in our clinical scoring system, which were dichotomized using cut-off points prior to score entry based on clinical experience and previously published evidence. We compared the diagnostic performance of different scores using the area under the receiver operating characteristic (AUCROC) curve. Bayesian decision theory was used to calculate the posterior probability of active cancer for different scores. All $P$ values were two-tailed. $P$ values $<0.05$ were considered statistically significant. 


\section{Results}

3.1. Baseline Characteristics of Patients with Ischemic Stroke in Both Groups. Of the initial 1,889 patients with IS, 799 were included in the final analysis (Figure 1). Of these, $53(6.63 \%)$ were diagnosed with active cancer at the time of stroke onset and were assigned to the cancer group, while 746 (93.37\%) did not have a history of cancer and were assigned to the control group.

Table 1 shows the patient characteristics for both groups. Patients in the cancer group had a significantly higher mean age than those without cancer (mean \pm SD: 67.21 \pm 10.11 years vs. $62.11 \pm 12.09$ years; $t=3.00, P<0.01$ ). The frequency of hyperlipidemia was lower in the cancer group than in the control group (22.6\% vs. $\left.49.3 \% ; \chi^{2}=14.13, P<0.01\right)$. The prevalence of other vascular risk factors did not differ significantly between the two groups. We observed that $35(66.04 \%)$ patients in the cancer group had SUE compared to 31 (4.16\%) patients in the control group $\left(\chi^{2}=250.06, P<0.01\right)$, while the LAA subtype was less common in the cancer group than in the control group ( $9.43 \%$ vs. $60.99 \% ; \chi^{2}=53.85, P<0.01$ ), as per the TOAST criteria. There were no significant differences in prevalence of the CE, SVO, and SOE subtypes. We found that the average NIHSS score at admission was higher in the cancer group than in the control group (mean $\pm \mathrm{SD}$ : $6.13 \pm 4.10$ vs. $4.30 \pm 4.48 ; t=2.89, P<0.01)$. AMCI were observed more frequently in the cancer group than in the control group (56.60\% vs. $19.84 \% ; \chi^{2}=38.63, P<0.01$ ). The incidence of deep vein thrombosis or pulmonary embolism during hospitalization was higher in the cancer group than in the control group ( $13.21 \%$ vs. $\left.3.75 \% ; \chi^{2}=10.56, P<0.01\right)$. Patients with active cancer had significantly higher serum levels of fibrinogen (mean \pm SD: $4.44 \pm 1.82 \mathrm{~g} / \mathrm{L}$ vs. $3.56 \pm 1.02 \mathrm{~g} / \mathrm{L} ; t=3.49$, $P<0.01$ ), D-dimer (median [IQR]: $2.23 \mathrm{mg} / \mathrm{mL}$ [0.98-10.76] vs. $0.35 \mathrm{mg} / \mathrm{mL}[0.27-0.48] ; Z=-9.55, P<0.01$ ), and CRP (median [IQR]: $18.40 \mathrm{mg} / \mathrm{L}$ [5.45-46.00] vs. $3.90 \mathrm{mg} / \mathrm{L}$ [2.80-6.33]; $Z=-7.35, P<0.01)$ but significantly lower levels of hemoglobin (mean \pm SD: $119.79 \pm 25.68 \mathrm{~g} / \mathrm{L}$ vs. $143.61 \pm$ $18.57 \mathrm{~g} / \mathrm{L} ; t=-6.63, P<0.01)$ than those without.

3.2. Type, Histology, and Stage of Active Cancer. The most commonly observed cancer diagnoses among patients with IS and active cancer were as follows: lung cancer $(n=16$, $30.19 \%)$; gastric cancer $(n=8,15.09 \%)$; liver cancer $(n=6$, $11.32 \%)$; colorectal cancer $(n=5,9.43 \%)$; breast cancer $(n=5,9.43 \%)$; genitourinary cancers (bladder, prostate, and ovarian cancers; $n=5,9.43 \%)$; biliary tract cancer $(n=4$, $7.55 \%)$; and pancreatic cancer, nasopharyngeal cancer, renal cancer, and adrenal cancer ( $n=1,1.89 \%$ each). Thirty-one $(58.49 \%)$ patients were diagnosed with adenocarcinoma, and $21(39.62 \%)$ were diagnosed with metastatic disease (Table 2).

3.3. Univariate and Multivariate Logistic Regression Analyses. We assessed all risk factors with $P$ values $\leq 0.05$ (Table 1 ) using a univariate logistic regression model (including age, hyperlipidemia, LAA, SUE, NIHSS score, AMCI, hemoglobin, fibrinogen, D-dimer, and CRP). The results are shown in Table 3. Notably, the odds ratios (OR) for age, NIHSS score, hemoglobin, and CRP were approximately 1 on uni- variate logistic regression analysis. Therefore, we considered only the remaining risk factors, including hyperlipidemia, SUE, AMCI, presence of venous thromboembolism or pulmonary embolism, and fibrinogen and D-dimer levels, in the multivariate logistic regression model. Multivariate analysis showed that the absence of a history of hyperlipidemia $(\mathrm{OR}=0.17,95 \%$ confidence interval $(\mathrm{CI}): 0.06-0.48, P$ $<0.01)$, elevated serum fibrinogen levels $(\mathrm{OR}=1.72,95 \%$ CI: $1.33-2.22, P<0.01)$, elevated $\mathrm{D}$-dimer levels $(\mathrm{OR}=1.43$ , 95\% CI 1.24-1.64, $P<0.01)$, and SUE (OR $=22.87,95 \%$ CI: 9.91-52.78, $P<0.01)$ were independently associated with active cancer (Table 3 ).

3.4. Development of a Predictive Score Using Areas under the Receiver Operating Characteristic Curves. We developed a scoring system to predict active cancer in patients with IS, based on the findings of multivariate analysis, particularly in the subset of patients with SUE. The final score, ranging from 0 to 3 , comprised the sum of individual scores for the history of hyperlipidemia, serum D-dimer levels, and serum fibrinogen levels. We reviewed and compared several studies to determine the appropriate cut-off values for D-dimer and fibrinogen levels for the scoring system. The OASIS-CANCER study conducted by Lee et al. determined the first quartile of the pretreatment D-dimer concentration as $2.08 \mu \mathrm{g} / \mathrm{mL}$ and the median fibrinogen concentration as $399 \mathrm{mg} / \mathrm{dL}$ [21]. Moreover, Quintas et al. found that the median fibrinogen level of patients with IS without cancer was $408.5 \mathrm{mg} / \mathrm{dL}$ [22]. They demonstrated that fibrinogen values were associated with the diagnosis of cancer after IS. Therefore, we analyzed multiple data conditions and assigned the following final scores, according to the findings of previous studies, our clinical experience, and the findings of this study: history of hyperlipidemia $=0$ points, no history of hyperlipidemia $=1$ point; $\mathrm{D}$-dimer level $\leq 2.00 \mu \mathrm{g} / \mathrm{mL}=0$ points, D-dimer level $>2.00 \mu \mathrm{g} / \mathrm{mL}=1$ point; and fibrinogen level $\leq 4.00 \mathrm{~g} / \mathrm{L}=0$ points, fibrinogen level $>4.00 \mathrm{~g} / \mathrm{L}=1$ point. Table 4 presents the sensitivity, specificity, and posterior probability of each cut-off point based on the supposed cancer prevalence value of $6.63 \%$ in our study. Figure 2 shows that the probability of active cancer was $59 \%$ if a patient with IS had a clinical score of 3 points, with a reliable AUC-ROC value of 0.83 (95\% CI: $0.77-0.89, P<0.01$ ).

\section{Discussion}

This study showed that the absence of a history of hyperlipidemia, increased serum levels of D-dimer and fibrinogen, and SUE were independent risk factors associated with active cancer in patients with IS. Adenocarcinoma was the most commonly observed histological manifestation, consistent with the results of several previous studies [21, 23, 24]. However, recent studies evaluating underlying cancer in patients with IS have simply focused on differences in epidemiological and biochemical parameters. There is no consensus on the optimal method or time for identifying occult cancer in patients with acute IS. Thus, in light of our findings, we developed a reliable predictive score that can help clinical neurologists to rapidly screen the possibility of occult cancer 


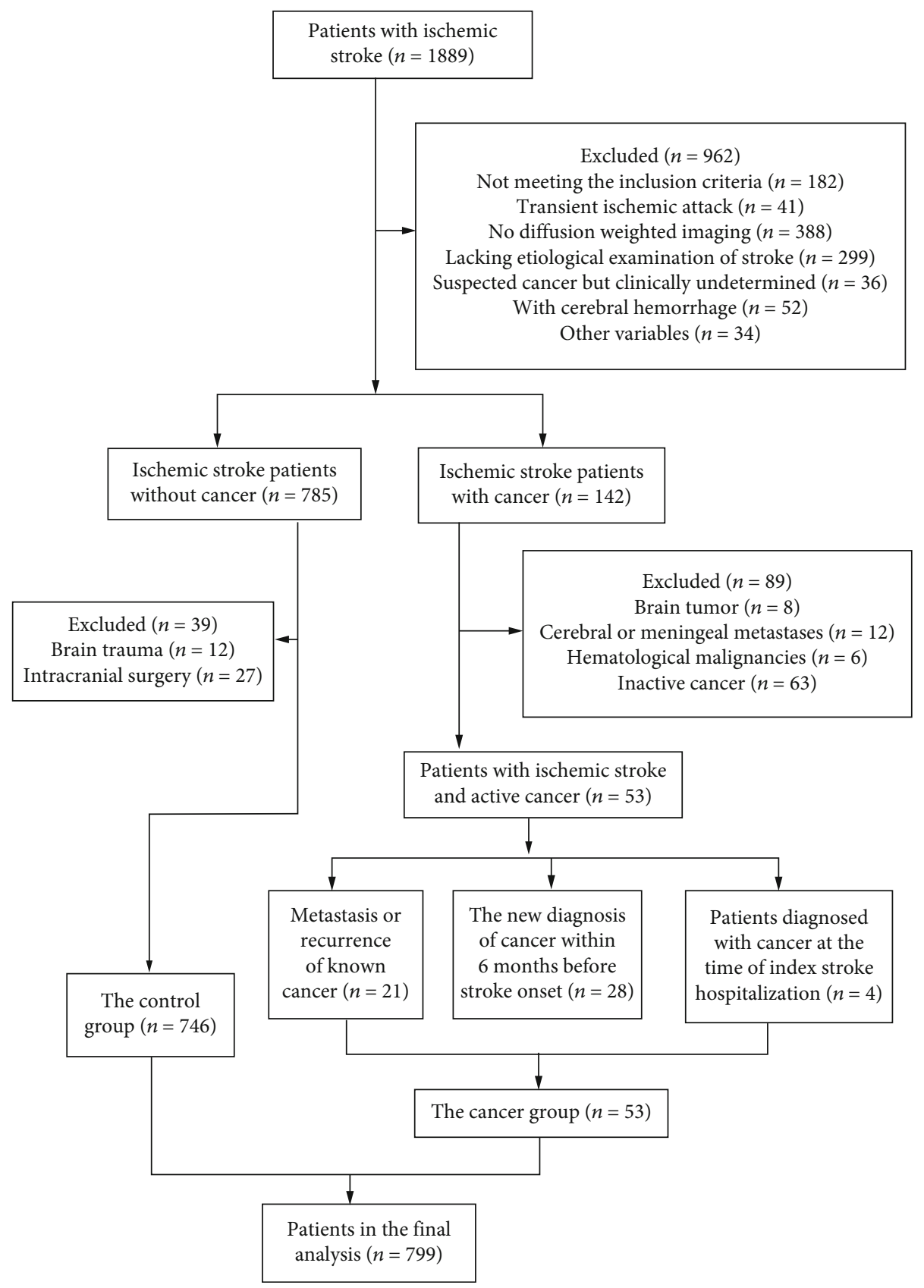

FIGURE 1: Flow diagram explaining the inclusion and exclusion criteria.

in patients with IS at an early stage, which could ultimately improve patients' quality of life and survival outcomes.

Our systematically developed predictive score consisted of increased serum levels of D-dimer and fibrinogen and the absence of a history of hyperlipidemia, especially in patients with SUE. Our final scoring system demonstrated that if a patient with IS had a total score of $3 / 3$, the probability of active cancer was 59\% (with high specificity [99\%]), based on an assumed cancer prevalence of $6.63 \%$, which was similar to that in previous studies $[22,25,26]$. The probability of active cancer was $27 \%$ if the score was 2 points. We compared the predictive capabilities of our scoring system with those of a previously reported system and found that our system was significantly superior; the AUC of the score described by Selvik et al. was 0.73 [15], while the AUC of our score was 0.83 .

The differences in classical vascular risk factors between patients with IS with and without cancer remain unclear. Some studies have reported that the prevalence of classical vascular risk factors was similar between these patient groups $[18,27,28]$, while others have demonstrated that the frequency of vascular risk factors, especially hyperlipidemia, was lower in patients with IS and active cancer $[29,30]$. Our analyses demonstrated that the absence of a history of hyperlipidemia was independently associated with the presence of active cancer in patients with IS. Previous studies have suggested that low total cholesterol levels were associated with 
TABLE 1: Comparison of baseline characteristics between patients with ischemic stroke and active cancer and those without cancer.

\begin{tabular}{|c|c|c|c|c|}
\hline Variables & Cancer group $n=53$ & Control group $n=746$ & $t / \chi^{2}$ & $P$ value \\
\hline \multicolumn{5}{|l|}{ Demographics } \\
\hline Age (years) & $67.21 \pm 10.11$ & $62.11 \pm 12.09$ & 3.00 & $<0.01$ \\
\hline Sex (\% male) & $34(64.2)$ & $522(70.0)$ & 0.79 & 0.37 \\
\hline \multicolumn{5}{|l|}{ Vascular risk factors } \\
\hline Hypertension & $30(56.6)$ & $508(68.1)$ & 2.97 & 0.09 \\
\hline Diabetes mellitus & $14(26.4)$ & $286(38.3)$ & 3.00 & 0.08 \\
\hline Hyperlipidemia & $12(22.6)$ & $368(49.3)$ & 14.13 & $<0.01$ \\
\hline Atrial fibrillation & $4(7.6)$ & $46(6.2)$ & & 0.57 \\
\hline Coronary heart disease & $8(15.1)$ & $106(14.2)$ & 0.03 & 0.86 \\
\hline Previous stroke/TIA & $7(13.2)$ & $129(17.3)$ & 0.59 & 0.45 \\
\hline Current smoking & $25(47.2)$ & $351(47.1)$ & 0.00 & 0.99 \\
\hline Obesity & $6(11.3)$ & $121(16.2)$ & 0.89 & 0.35 \\
\hline \multicolumn{5}{|l|}{ TOAST classification } \\
\hline Large-artery atherosclerosis & $5(9.4)$ & $455(61.0)$ & 53.85 & $<0.01$ \\
\hline Cardioembolism & $2(3.8)$ & $33(4.4)$ & & 1.00 \\
\hline Small vessel occlusion & $9(17.0)$ & $216(29.0)$ & 3.51 & 0.06 \\
\hline Other determined etiology & $2(3.8)$ & $11(1.5)$ & & 0.21 \\
\hline Undetermined etiology & $35(66.0)$ & $31(4.2)$ & 250.6 & $<0.01$ \\
\hline \multicolumn{5}{|l|}{ Ischemic stroke characteristics } \\
\hline Initial NIHSS score & $6.13 \pm 4.10$ & $4.30 \pm 4.48$ & 2.89 & $<0.01$ \\
\hline AMCI & $30(56.6)$ & $148(19.8)$ & 38.63 & $<0.01$ \\
\hline VTE or PE & $7(13.2)$ & $28(3.8)$ & 10.56 & $<0.01$ \\
\hline \multicolumn{5}{|l|}{ Laboratory markers } \\
\hline Hemoglobin (g/L) & $119.79 \pm 25.68$ & $143.61 \pm 18.58$ & -6.63 & $<0.01$ \\
\hline Platelets $\left(/ 10^{9}\right)$ & $213.28 \pm 99.86$ & $222.17 \pm 66.55$ & -0.64 & 0.53 \\
\hline Fibrinogen $(\mathrm{g} / \mathrm{L})$ & $4.44 \pm 1.82$ & $3.56 \pm 1.02$ & 3.49 & $<0.01$ \\
\hline D-dimer (mg/mL) & $2.23(0.98-10.76)$ & $0.35(0.27-0.48)$ & -9.55 & $<0.01$ \\
\hline CRP (mg/L) & $18.40(5.45-46.00)$ & $3.90(2.80-6.33)$ & -7.35 & $<0.01$ \\
\hline
\end{tabular}

Data are presented as the mean \pm standard deviation, median (interquartile range), or $n$ (\%). Abbreviations: AMCI: acute multiple cerebral infarctions; VTE: venous thromboembolism; PE: pulmonary embolism; CRP: C-reactive protein; NIHSS: National Institute of Health Stroke Scale; TOAST: Trial of Org 10172 in Acute Stroke Treatment; TIA: transient ischemic attack.

an elevated risk of cancer incidence and mortality [31-33], and this inverse association has been well demonstrated in patients with stomach, breast, and prostate cancers [32, 34]. The findings of these studies are generally in accordance with those of our study. Moreover, we found a significant difference in the classification of stroke etiology between the two groups. Similar to previous studies, we observed a lower frequency of the LAA subtype and a higher prevalence of SUE in patients with IS and active cancer than in those without cancer [12, 29]. Thus, previous studies and our study indicate that a specific stroke mechanism, differing from that associated with traditional IS, may exist in patients with active cancer.

We compared the differences in blood coagulation biomarkers between the two groups, including an analysis of platelet, fibrinogen, and D-dimer levels to further explore the possible mechanisms underlying cancer-associated IS. We found higher serum levels of fibrinogen and D-dimer in patients with IS and active cancer than in those without cancer, similar to most recent studies $[11,21,22]$. These factors are even considered to be predictors of active cancer in patients with IS [35]. Earlier studies have reported that Ddimer levels, which reflect excessive fibrin turnover associated with an activated coagulation system, were correlated with the degree of hypercoagulability [21] and widespread dissemination of microthrombi [36]. Moreover, malignancies can induce a hypercoagulable state and promote the formation of microthrombi by promoting the secretion of mucins, release of tissue factors, and production of procoagulant cytokines [37], which may explain the elevated levels of blood coagulation biomarkers in the present study. Therefore, it is necessary to identify the specific cancer types that tend to induce hypercoagulable states and thrombosis. Our data demonstrated that active lung cancer and gastric cancer were the most common cancer types in IS patients, and adenocarcinoma was the most commonly observed histopathological subtype. These findings are also consistent with 
TABLE 2: Characteristics of patients with ischemic stroke and active cancer.

\begin{tabular}{lcc}
\hline Cancer location & Histological type & Number \\
\hline Lung cancer & Adenocarcinoma & 15 \\
& Small cell carcinoma & 1 \\
Gastric cancer & Adenocarcinoma & 8 \\
Liver cancer & Hepatocellular carcinoma & 6 \\
Colon cancer & Adenocarcinoma & 3 \\
Rectal cancer & Adenocarcinoma & 2 \\
Breast cancer & Infiltrating ductal carcinoma & 4 \\
& Medullary carcinoma & 1 \\
Biliary tract cancer & Epithelial cell carcinoma & 3 \\
Bladder cancer & Adenocarcinoma & 1 \\
Prostate cancer & Transitional epithelial carcinoma \\
Ovarian cancer & Adenocarcinoma \\
Pancreatic cancer & Serous carcinoma \\
Nasopharyngeal cancer & Adenocarcinoma \\
Renal cancer & Squamous cell carcinoma \\
Adrenal cancer & Clear cell carcinoma \\
Metastatic disease & Cortical carcinoma \\
\hline
\end{tabular}

TABLE 3: Univariate and multivariate logistic regression analyses of risk factors in patients with ischemic stroke with active cancer and those without cancer.

\begin{tabular}{lccc}
\hline Variables & \multicolumn{2}{c}{ Univariate logistic regression analysis } & \multicolumn{2}{c}{$\begin{array}{c}\text { Multivariate logistic regression analysis } \\
\text { OR }(95 \% \text { CI })\end{array}$} & \\
\hline Age & OR $(95 \% \mathrm{CI})$ & $<0.01$ & $0.16(0.06-0.45)$ \\
Hyperlipidemia & $1.04(1.01-1.07)$ & $<0.01$ & \\
LAA & $0.30(0.16-0.58)$ & $<0.01$ & $<0.30(7.93-48.96)$ \\
SUE & $0.07(0.02-0.17)$ & $<0.01$ & $<0.01$ \\
NIHSS score & $44.85(22.89-87.88)$ & $<0.01$ & $1.58(0.66-3.81)$ \\
AMCI & $1.06(1.02-1.11)$ & $<0.01$ & $0.92(0.21-3.94)$ \\
VTE or PE & $5.27(2.97-9.34)$ & $<0.01$ & 0.31 \\
Hemoglobin $(\mathrm{g} / \mathrm{L})$ & $4.56(1.97-10.58)$ & $<0.01$ & $1.70(1.31-2.21)$ \\
Fibrinogen $(\mathrm{g} / \mathrm{L})$ & $0.95(0.94-0.96)$ & $<0.01$ & $1.42(1.23-1.65)$ \\
D-dimer $(\mu \mathrm{g} / \mathrm{mL})$ & $1.63(1.35-2.00)$ & $<0.01$ & $<0.01$ \\
CRP & $1.72(1.45-2.03)$ & $<0.01$ & $<0.01$ \\
\hline
\end{tabular}

LAA: large-artery atherosclerosis; SUE: stroke of undetermined etiology; NIHSS: National Institute of Health Stroke Scale; AMCI: acute multiple cerebral infarctions; VTE: venous thromboembolism; PE: pulmonary embolism; CRP: C-reactive protein.

TABle 4: Performance and posterior probabilities of the predictive score for cancer in patients with ischemic stroke.

\begin{tabular}{lccc}
\hline Score & Sensitivity & Specificity & Posterior probability \\
\hline 1 & 0.96 & 0.37 & 0.09 \\
2 & 0.68 & 0.88 & 0.27 \\
3 & 0.19 & 0.99 & 0.59 \\
\hline
\end{tabular}

those of previous studies, which revealed a higher prevalence of thromboembolic events in patients with lung cancer and adenocarcinoma [25, 38, 39]. Furthermore, studies have shown that patients with adenocarcinoma were prone to developing cancer-mediated hypercoagulability and microemboli $[18,40]$. Several studies have suggested that some epithelium-derived tumors, such as those originating from the lung, stomach, and bile ducts, were frequently adenocarcinomas and could systematically secrete mucins that bind to Pand L-selectins, inducing the formation of platelet-rich microthrombi [41, 42]. Moreover, these cancers usually remain undiagnosed until they reach an advanced or metastatic stage. The histologic and natural characteristics of these tumors underline the potential increase in the risk of IS, further revealing the role of hypercoagulability in cancer-related IS.

Our study has several limitations. First, this was a singlecenter retrospective study performed using consecutively 


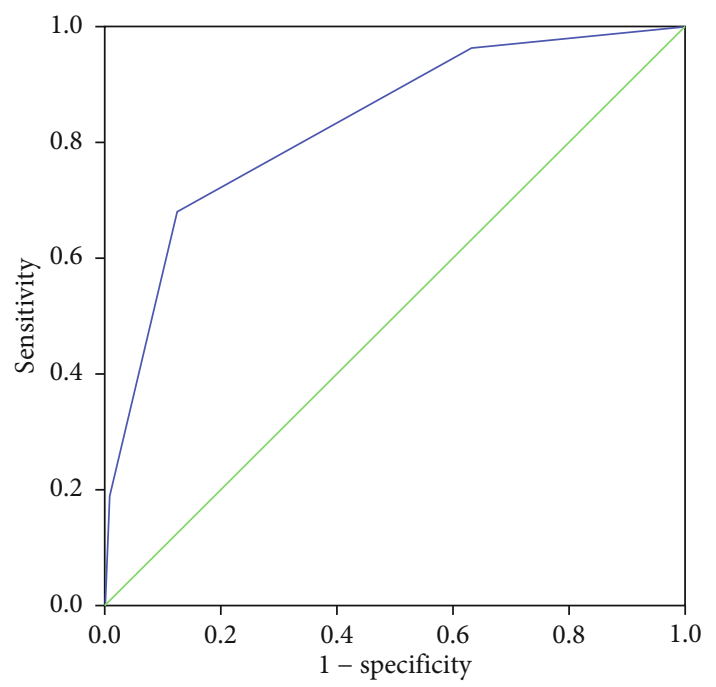

FIgURE 2: Receiver operating characteristic curves of the clinical scores. The final scores were as follows: history of hyperlipidemia $=0$ points, no history of hyperlipidemia $=1$ point; $\mathrm{D}$-dimer level $\leq 2.00 \mu \mathrm{g} / \mathrm{mL}=0$ points, D-dimer level $>2.00 \mu \mathrm{g} / \mathrm{mL}=1$ point; and fibrinogen level $\leq 4.00 \mathrm{~g} / \mathrm{L}=0$ points, fibrinogen level $>4.00 \mathrm{~g} /$ $\mathrm{L}=1$ point.

collected data. Therefore, our findings may not be applicable to other settings due to the inherent selection bias. Second, comparing the characteristics of patients according to different cancer types would have been ideal, as patients with IS may have different types of cancer. However, further categorization by cancer type did not allow for appropriate subgroup analyses, because this study included a relatively limited number of patients with active cancer. Third, we could not clarify the relationship between the value of hypercoagulation biomarkers and tumor markers, as this is difficult to achieve for a retrospective study; furthermore, tumor markers were not routinely tested in the Department of Neurology due to Chinese national conditions and the high cost of examination.

\section{Conclusions}

This study systematically developed a scoring system comprising the history of hyperlipidemia and fibrinogen and Ddimer levels for the prediction of active cancer in patients with IS, especially in those with SUE. Our findings indicate the importance of hypercoagulability in assessing active cancer in patients with IS, which could support early decisionmaking for intervention and management at the time of admission. Prospective multicenter studies are needed to evaluate the effectiveness of this clinical scoring system prior to implementation in clinical practice.

\section{Data Availability}

Data has not been made accessible in the interest of protecting patients' privacy.

\section{Ethical Approval}

This study was performed in accordance with the principles of the Declaration of Helsinki. The Medical Science Research Ethics Committee of the First Affiliated Hospital of China Medical University approved this study (committee's reference number [2019]325).

\section{Consent}

The need for informed consent (prior to participation) was waived because of the retrospective nature of this study and the minimal risk posed to patients in this study.

\section{Conflicts of Interest}

The authors have no competing interests to declare.

\section{Authors' Contributions}

Jiwei Jiang contributed in conceptualization and design of the study, interpretation of data, and drafting and revising the manuscript. Xiuli Shang contributed in conceptualization and critical revision of the manuscript. Jinming Zhao contributed in data collection and analyses and ethics submission. Meihui Cao contributed in data analyses and data collection. Jirui Wang contributed in data analyses. Runzhi Li contributed in data analyses. Yanli Wang contributed in data analyses. Jun Xu contributed in critical revision of the manuscript for important intellectual content, study supervision, and funding support.

\section{Acknowledgments}

This study was supported by the National Natural Science Foundation of China (81870821 and 82071187) and Beijing Youth Talent Team Support Program (2018000021223TD08), both of which were from the corresponding author Jun $\mathrm{Xu}$. The funding body provided funding for data collection, analysis, and interpretation, as well as writing of the manuscript. We would like to thank Editage (http://www.editage.cn) for English language editing.

\section{References}

[1] GBD 2013 Mortality and Causes of Death Collaborators, "Global, regional, and national age-sex specific all-cause and cause-specific mortality for 240 causes of death, 1990-2013: a systematic analysis for the Global Burden of Disease Study 2013," The Lancet, vol. 385, pp. 117-171, 2015.

[2] W. Grisold, S. Oberndorfer, and W. Struhal, "Stroke and cancer: a review," Acta Neurologica Scandinavica, vol. 119, no. 1, pp. 1-16, 2009.

[3] N. Sanossian, C. Djabiras, W. J. Mack, and B. Ovbiagele, "Trends in cancer diagnoses among inpatients hospitalized with stroke," Journal of Stroke and Cerebrovascular Diseases, vol. 22, no. 7, pp. 1146-1150, 2013.

[4] B. B. Navi, A. S. Reiner, H. Kamel et al., "Association between incident cancer and subsequent stroke," Annals of Neurology, vol. 77, no. 2, pp. 291-300, 2015. 
[5] B. Rioux, L. Touma, A. Nehme, G. Gore, M. R. Keezer, and L. C. Gioia, "Frequency and predictors of occult cancer in ischemic stroke: a systematic review and meta-analysis," International Journal of Stroke, vol. 16, no. 1, pp. 12-19, 2021.

[6] R. A. Salazar-Camelo, E. A. Moreno-Vargas, A. F. Cardona, and H. F. Bayona-Ortiz, "Ischemic stroke: a paradoxical manifestation of cancer," Critical Reviews in Oncology/Hematology, vol. 157, p. 103181, 2021.

[7] H. H. G. Castro, A. P. Alencar, I. M. Benseñor, P. A. Lotufo, and A. C. Goulart, "Multimorbidities are associated to lower survival in ischaemic stroke: results from a Brazilian Stroke Cohort (EMMA study)," Cerebrovascular Diseases, vol. 44, no. 3-4, pp. 232-239, 2017.

[8] J. L. Dearborn, V. C. Urrutia, and S. R. Zeiler, "Stroke and cancer-a complicated relationship," Journal of Neurology \& Translational Neuroscience, vol. 2, p. 1039, 2015.

[9] J.-y. Wang, G.-j. Zhang, S.-x. Zhuo et al., "D-dimer $>2.785 \mu \mathrm{g} / \mathrm{ml}$ and multiple infarcts $\geq 3$ vascular territories are two characteristics of identifying cancer-associated ischemic stroke patients," Neurological Research, vol. 40, no. 11, pp. 948-954, 2018.

[10] Y. Gon, M. Sakaguchi, J. Takasugi et al., "Plasma D-dimer levels and ischaemic lesions in multiple vascular regions can predict occult cancer in patients with cryptogenic stroke," European Journal of Neurology, vol. 24, no. 3, pp. 503-508, 2017.

[11] A. G. Karlińska, G. Gromadzka, M. A. Karliński, and A. Członkowska, "The activity of malignancy may determine stroke pattern in cancer patients," Journal of Stroke and Cerebrovascular Diseases, vol. 24, pp. 348-353, 2015.

[12] S. G. Kim, J. M. Hong, H. Y. Kim et al., "Ischemic stroke in cancer patients with and without conventional mechanisms: a multicenter study in Korea," Stroke, vol. 41, no. 4, pp. 798801,2010

[13] D. M. Cestari, D. M. Weine, K. S. Panageas, A. Z. Segal, and L. M. DeAngelis, "Stroke in patients with cancer: incidence and etiology," Neurology, vol. 62, no. 11, pp. 2025-2030, 2004.

[14] Y. Y. Zhang, D. Cordato, Q. Shen, A. Z. Sheng, W. T. Hung, and D. K. Chan, "Risk factor, pattern, etiology and outcome in ischemic stroke patients with cancer: a nested case-control study," Cerebrovascular Diseases, vol. 23, no. 2-3, pp. 181187, 2007.

[15] H. A. Selvik, A. T. Bjerkreim, L. Thomassen, U. WajeAndreassen, H. Naess, and C. E. Kvistad, "When to screen ischaemic stroke patients for cancer," Cerebrovascular Diseases, vol. 45, no. 1-2, pp. 42-47, 2018.

[16] C. J. Johnson, S. J. Kittner, R. J. McCarter et al., "Interrater reliability of an etiologic classification of ischemic stroke," Stroke, vol. 26, no. 1, pp. 46-51, 1995.

[17] J. Yoo, H. S. Nam, Y. D. Kim, H. S. Lee, and J. H. Heo, "Shortterm outcome of ischemic stroke patients with systemic malignancy," Stroke, vol. 50, no. 2, pp. 507-511, 2019.

[18] B. B. Navi, S. Singer, A. E. Merkler et al., "Recurrent thromboembolic events after ischemic stroke in patients with cancer," Neurology, vol. 83, no. 1, pp. 26-33, 2014.

[19] L. Goldstein and G. Samsa, "Reliability of the national institutes of health stroke scale," Stroke, vol. 28 , no. 2, pp. $307-$ 310, 1997.

[20] H. P. Adams, B. H. Bendixen, L. J. Kappelle et al., "Classification of subtype of acute ischemic stroke. Definitions for use in a multicenter clinical trial. TOAST. Trial of Org 10172 in acute stroke treatment," Stroke, vol. 24, no. 1, pp. 35-41, 1993.

[21] M. J. Lee, J. W. Chung, M. J. Ahn et al., "Hypercoagulability and mortality of patients with stroke and active cancer: the OASIS-CANCER study," J Stroke., vol. 19, no. 1, pp. 77-87, 2017.

[22] S. Quintas, J. Rogado, P. Gullón et al., "Predictors of unknown cancer in patients with ischemic stroke," Journal of NeuroOncology, vol. 137, no. 3, pp. 551-557, 2018.

[23] K. W. Nam, C. K. Kim, T. J. Kim et al., "D-dimer as a predictor of early neurologic deterioration in cryptogenic stroke with active cancer," European Journal of Neurology, vol. 24, no. 1, pp. 205-211, 2017.

[24] S. Grazioli, M. Paciaroni, G. Agnelli et al., "Cancer-associated ischemic stroke: a retrospective multicentre cohort study," Thrombosis Research, vol. 165, pp. 33-37, 2018.

[25] H. A. Selvik, L. Thomassen, A. T. Bjerkreim, and H. Næss, "Cancer-associated stroke: the Bergen NORSTROKE study," Cerebrovasc Dis Extra., vol. 5, no. 3, pp. 107-113, 2015.

[26] M. H. Sorgun, M. Kuzu, I. S. Ozer et al., "Risk factors, biomarkers, etiology, outcome and prognosis of ischemic stroke in cancer patients," Asian Pacific Journal of Cancer Prevention, vol. 19, no. 3, pp. 649-653, 2018.

[27] B. Sun, S. Fan, Z. Li et al., "Clinical and neuroimaging features of acute ischemic stroke in cancer patients," European Neurology, vol. 75, no. 5-6, pp. 292-299, 2016.

[28] P. Corraini, A. G. Ording, V. W. Henderson, S. Szépligeti, E. Horváth-Puhó, and H. T. Sørensen, "Cancer, other comorbidity, and risk of venous thromboembolism after stroke: a population-based cohort study," Thrombosis Research, vol. 147, pp. 88-93, 2016.

[29] S. Oberndorfer, V. Nussgruber, O. Berger, H. Lahrmann, and W. Grisold, "Stroke in cancer patients: a risk factor analysis," Journal of Neuro-Oncology, vol. 94, pp. 221-226, 2009.

[30] A. Carrilho Romeiro, A. Valadas, and J. Marques, "Acute ischemic stroke on cancer patients, a distinct etiology? A case-control study," Acta Médica Portuguesa, vol. 28, no. 5, pp. 613-618, 2015.

[31] M. Li, J. Lu, J. Fu et al., "The association and joint effect of serum cholesterol, glycemic status with the risk of incident cancer among middle-aged and elderly population in China cardiometabolic disease and cancer cohort (4C)-study," American Journal of Cancer Research, vol. 10, no. 3, pp. 975-986, 2020.

[32] V. A. Katzke, D. Sookthai, T. Johnson, T. Kühn, and R. Kaaks, "Blood lipids and lipoproteins in relation to incidence and mortality risks for CVD and cancer in the prospective EPICHeidelberg cohort," BMC Medicine, vol. 15, no. 1, p. 218, 2017.

[33] B. Wu, L. Teng, D. He, D. D. Yu, and F. Jiang, "Dose-response relation between serum total cholesterol levels and overall cancer risk: evidence from 12 prospective studies involving 1,926,275 participants," International Journal of Food Sciences and Nutrition, vol. 70, no. 4, pp. 432-441, 2019.

[34] K. Asano, M. Kubo, K. Yonemoto et al., "Impact of serum total cholesterol on the incidence of gastric cancer in a populationbased prospective study: the Hisayama study," International Journal of Cancer, vol. 122, no. 4, pp. 909-914, 2008.

[35] Y. J. Guo, M. H. Chang, P. L. Chen, Y. S. Lee, Y. C. Chang, and Y. C. Liao, "Predictive value of plasma (D)-dimer levels for cancer related stroke: a 3-year retrospective study," Journal of 
Stroke and Cerebrovascular Diseases, vol. 23, no. 4, pp. e249e254, 2014.

[36] J. M. Seok, S. G. Kim, J. W. Kim et al., "Coagulopathy and embolic signal in cancer patients with ischemic stroke," Annals of Neurology, vol. 68, no. 2, pp. 213-219, 2010.

[37] A. Varki, “Trousseau's syndrome: multiple definitions and multiple mechanisms," Blood, vol. 110, no. 6, pp. 1723-1729, 2007.

[38] R. Kassubek, L. Bullinger, J. Kassubek et al., "Identifying ischemic stroke associated with cancer: a multiple model derived from a case-control analysis," Journal of Neurology, vol. 264, no. 4, pp. 781-791, 2017.

[39] J. Lin, S. Wu, R. Xu et al., "Clinical characteristics and risk factors of lung cancer-associated acute ischemic stroke," BioMed Research International, vol. 2019, Article ID 6021037, 7 pages, 2019.

[40] E. J. Lee, H. W. Nah, J. Y. Kwon, D. W. Kang, S. U. Kwon, and J. S. Kim, "Ischemic stroke in patients with cancer: is it different from usual strokes?," International Journal of Stroke, vol. 9, no. 4, pp. 406-412, 2014.

[41] L. E. Neilson, L. R. Rogers, and S. Sundararajan, "Evaluation and treatment of a patient with recurrent stroke in the setting of active malignancy," Stroke, vol. 50, 2019.

[42] R. Bhatia, S. K. Gautam, A. Cannon et al., "Cancer-associated mucins: role in immune modulation and metastasis," Cancer Metastasis Reviews, vol. 38, no. 1-2, pp. 223-236, 2019. 